\title{
Être aux anges, Sortir de ses gonds... Comment les langues traduisent-elles des états émotionnels ?
}

\author{
Céline Vaguer \\ Université de Toulouse II-Le Mirail - CNRS UMR 5263 (CLLE-ERSS) \\ vaguer@univ-tlse2.fr
}

De par la diversité des langues parlées dans le monde (entre 6000 et 7000 langues) et la généralisation des échanges, la traduction, et en particulier la traduction automatique, répond aujourd'hui à des enjeux économiques ${ }^{1}$, politiques, culturels, techniques, scientifiques qui s'illustrent par une diffusion de produits sur différents supports : traduction de textes officiels comme de logiciels, de jeux vidéo, de sous-titres de films, de posologies de médicaments, de modes d'emploi, de prospectus publicitaires, de romans, etc. Autrement dit, tout est susceptible d'être traduit.

Si pour le linguiste toutes les langues se valent (Hagège 1987, Calvet 1999, Van Hoof 2009, entre autres), comment doit-il se positionner au regard de ces attentes (produire vite, bien et à moindre coût) et peut-il y répondre ?

Rappelons que l'objectif du linguiste est d'étudier de manière objective, descriptive et explicative la structure des langues. Selon le point de vue adopté par le linguiste (diachronique vs synchronique), l'étude d'une seule et même langue - un état de langue - se focalise soit sur son fonctionnement (les éléments et facteurs mis en jeu) à un moment déterminé de son histoire (perspective synchronique), soit sur son évolution dans le temps (perspective diachronique qui étudie les différents états de développement d'une langue). L'un n'excluant pas l'autre. Quel que soit le point de vue retenu, le linguiste aura à cœur d'étudier la langue (par exemple, le français) par l'intermédiaire de chacune des unités qui la constituent: l'étude des sons sous l'angle de leur émission, caractères physiques (combinatoire, acoustique, articulation) et réception (la phonétique) ; l'étude des sons en tant qu'ils ont une fonction distinctive dans le système de la langue (la phonologie); l'étude de la forme des mots (la morphologie) ; l'étude des combinaisons de mots en groupes et en phrases (la syntaxe); l'étude du sens des unités lexicales (la lexicologie); l'étude systématique du sens des unités significatives (morphèmes, mots) et/ou de leurs combinaisons en syntagmes et en phrases (la sémantique), etc. - ou d'étudier les langues dans leur diversité : classification des langues (perspective comparatiste), comparaison terme à terme, rigoureuse et systématique, de la structure des langues (linguistique contrastive), étude du transfert (processus cognitif et/ou résultat) de textes d'une langue à l'autre (la traductologie), etc.

Le linguiste, par les descriptions qu'il propose, peut répondre aux attentes économiques et technologiques énoncées supra. Nous le démontrerons par le contenu même de notre contribution : l'étude des expressions figées dénotant des états émotionnels telles que être aux anges, sortir de ses gonds... et leur traduction en anglais et en espagnol. L'évaluation de logiciels de traduction automatique révèlera que la technologie ne peut être compétitive si elle ne tient pas compte des études descriptives réalisées par les linguistes.

« En tant qu'activité de service, la traduction génère un chiffre d'affaires important [...]. On estimait en 1997 le chiffre d'affaires de la traduction et de l'interprétation sur le marché libre à 3,75 milliard d'euros. Quatre ans plus tard, on peut estimer que ce chiffre d'affaires a pratiquement doublé » (Gouadec, 2002 : enjeux de la traduction). 


\section{Les expressions figées : langue et culture ${ }^{2}$}

\subsection{Pourquoi travailler sur les expressions figées?}

L'usage des expressions figées est loin d'être un phénomène marginal dans les langues: Maurice Gross (1982 \& 1988) dit en avoir listé plus de 40000 rien que pour le français (ce que confirme François Bercker (1994 \& 1999) ${ }^{3}$ ), ce qui contredit la conception traditionnelle qui les considère comme des exceptions - «ignorer ces constructions revient à ignorer une bonne partie du langage " (Danlos 1981). Mais du fait de cette fréquence, un traducteur ou un apprenant peuvent rester sans voix : les expressions figées " sont source de nombreuses difficultés lors de l'apprentissage d'une langue étrangère ou lors d'une traduction (quelle soit manuelle ou automatique). Néanmoins, elles ne font l'objet que de quelques dictionnaires bilingues sans prétention scientifique, et elles ne sont pas au centre d'études de linguistique comparée. II serait donc du plus haut intérêt que des lexiques-grammaires bilingues d'expressions figées soient développés " (Danlos 1988). A notre connaissance, il n'existe pas d'études accessibles ayant donné lieu à des lexiques bilingues exhaustifs d'expressions figées. Des études existent mais elles restent focalisées soit sur un certain type d'expression (par exemple celles contenant des parties du corps, des numéros ${ }^{4}$ ), soit sur des paires de langue (français/malgache, français/italien, français/anglais ${ }^{5}$ ). II est un fait que la France est toujours en retard dans le secteur technologique et que la recherche linguistique paraît inutile aux yeux des décideurs ${ }^{6}$.

\subsection{Expressions figées et culture}

Une fois mis en évidence la nécessité de travailler sur les expressions figées du fait de leur fréquence en langue, se pose la question de leur rapport à la culture. En effet, si les expressions figées reflètent la culture, comment prendre en compte cette dimension pour une « meilleure traduction" ?

\subsubsection{Figement : phénomène universel et culturel}

La lecture de travaux consacrés au figement montre l'existence de deux types d'approches du figement : l'approche 'linguistique' et l'approche 'culturelle'.

(a) l'approche 'linguistique' ne fait qu'évoquer le figement dans sa dimension culturelle, son but étant d'atteindre la caractérisation du figement sur le plan syntaxique,

2 Ce thème est également abordé dans $C$. Vaguer (à par.). Ce paragraphe s'en inspire donc profondément.

3 F. Bercker (1994 \& 1999) confirme les chiffres avancés par M. Gross et met en évidence que le champ sémantique des noms de parties du corps est le premier à entrer dans la composition d'expressions figées.

4 Par exemple, les expressions contenant des numéros (Cortès 2004), des noms de partie du corps (Bercker 1994 ; Martins-Baltar \& Calbris 1997 ; Valli \& Vilagines Serra 1998), entre autres.

5 Français/malgache (Andrienierenana et al. 2000), français/français du Québec (Labelle 1988), français/italien (Dugas \& Di-Sciullo 1978 ; Conenna 1985 ; Bidaud 2002), français/anglais (Billaudeau 1903 ; Dubois 1973), français/espagnol (Dugas \& Di-Sciullo 1978 ; Blanco 1998 \& 2001).

6 Alors même que «la démarche d'intelligence économique est particulièrement concernée par le développement de ces outils [les outils de traduction automatique], et ce à plusieurs égards : Dans ce secteur technologique de plus en plus porteur, les acteurs français, performants dans ces technologies nouvelles, et qui connaissent un développement rapide, sont aussi fragiles à cause de leur taille réduite et de leur dépendance à l'égard des grands donneurs d'ordre [...]. La langue étant un vecteur majeur pour le développement des normes, des concepts et des produits, il est important, pour l'influence et la compétitivité de la France, de développer la place du français dans l'usage de ce type d'outils [...]. Or, il apparaît, malgré le niveau élevé de la recherche française et des initiatives importantes telles que celle de 'Technolangue', que la France ne pourrait améliorer ses performances dans ce domaine que par un effort de l'Etat à la hauteur de celui consenti par d'autres pays [...] » (source: site Internet ministériel de l'intelligence économique). 
distributionnel et sémantique. On peut citer les études de G. Gougenheim (1971) ; M. Gross (1982, 1985, 1986, 1988 \& 1993); J. Giry (1984); L. Danlos (1988); J. Labelle (1988) ; G. Gross (1996) ; D. Gaatone (1997), J. Dubois \& F. Dubois-Charlier (2004), entre autres.

Ainsi, la préoccupation pour sa dimension culturelle et son étude constrastive, en vue de sa prise en charge par les logiciels de traitement de la langue, reste marginale (cf. entre autres E. Laporte (1988), S. Mejri (2001), C. Vaguer (2005, 2006 \& à par.)). L'aspect culturel n'est donc mentionné que sommairement : "dans les dictionnaires, on donne des exemples de collocations ou de phraséologie car il peut s'y nicher des éléments culturels" (Humbley 2004).

(b) l'approche 'culturelle', comme l'expose S. Mejri (1997), se pose la question du figement tant du point de vue de ce qu'il révèle de propre à une communauté donnée que du point de vue de l'universel (commun à toutes les communautés). Le figement peut en effet être perçu comme un processus certes universel (puisqu'on le retrouve dans beaucoup de langues) mais qui se singularise dans chaque langue: "si le figement [...] est un phénomène universel impliquant les mêmes mécanismes linguistiques et présentant plusieurs caractéristiques communes telles que la polylexicalité, la globalisation, la conceptualisation, la figuration, etc., il donne lieu dans chaque langue à des SF [séquences figées] propres : les parcours et les transferts de domaines, et les sélections sémiques sont rarement les mêmes " (Mejri 1997). Comment faire, donc, pour "dissocier le propre d'une communauté linguistique, le culturel, de ce qui est partagé par tous les humains, l'universel ? " (Ibid.).

Si l'on admet que les locuteurs de différentes langues organisent des réseaux semblables de phénomènes langagiers, par exemple des métaphores axées sur des parties du corps, comment se fait-il que "les expressions obtenues dans chacune des langues apparentées apparaissent sémantiquement opaques et posent des problèmes de traduction, compte tenu de la proximité des cultures, d'une histoire européenne largement partagée et d'environnements voisins" ? (Valli \& Vilagines Serra 1998). Cette interpellation met bien en évidence que le figement en tant que phénomène universel reste singulier, propre à chaque langue même si l'on peut établir entre les langues des rapprochements syntaxique, distributionnel et sémantique. Mais on ne sait comment s'opère cette singularisation, ce qui caractérise la culture du figement, la culture vue à travers le figement ${ }^{7}$.

\subsubsection{Figement : interdépendance de la langue et de la culture}

Maîtriser une langue, c'est maîtriser une culture et cela passe nécessairement par la maîtrise des expressions figées. Cet apprentissage culturel va bien au-delà des connaissances linguistiques, traditionnellement constituées de la phonologie, la syntaxe et le lexique et ne peut se réduire à un simple inventaire lexical. Le locuteur natif ne se rend pas toujours compte de l'idiomaticité de sa langue maternelle, ce qui peut provoquer des erreurs de traduction. Pour preuve, cette expérience relatée par F. J. Hausmann (1997) :

" une jeune Française qui fait son premier séjour en Allemagne [...] traduit mot à mot en allemand j'ai mal au cour, ne se doutant pas une seconde que le coeur n'est peut-être

\footnotetext{
Des études comparées devraient nous y aider en montrant notamment s'il existe ou non des correspondances entre les locutions dans les différences langues. $S^{\prime}$ il n'en existe pas, il faudra alors recourir à une analyse de la situation d'emploi pour pouvoir proposer une traduction adéquate. Ces études comparées permettraient également d'observer le niveau d'homologie entre les langues : soit la structure est identique (avoir des fourmis dans les jambes > sentir hormigueo en las piernas), soit partiellement identique (verbe, déterminant ou nom diffèrent, par exemple avoir un chat dans la gorge > to have a frog in the throat, avoir des fourmis dans les jambes > to have pins and needles in one's legs), soit tout est différent (pédaler dans la semoule > to be all at sea, mear fuera del tiesto)...
} 
pas le siège de la digestion dans l'optique des Allemands ou de la langue allemande. Elle ne sera d'ailleurs pas à un malentendu près, puisque le mot allemand que les dictionnaires donnent comme équivalent de digestion fait plutôt penser à l'intestin qu'à l'estomac et que le fameux foie français (j'ai le foie patraque / une crise de foie / mon foie, connais pas) n'a rien à voir avec cet organe et doit par conséquent se traduire tout autrement [...] Cela nous est parfois révélé par le comportement des dictionnaires qui ignorent certaines locutions ou, s'ils les recensent, se dispensent de les expliquer ".

Cette expérience - et les erreurs de traduction qui en découlent - révèle la place primordiale que revêt le culturel du point de vue de la traduction mais aussi l'inconnaissance par le locuteur des relations intimes qui lient la langue et la culture car il manque à l'apprenti traducteur "les données de culture générale liées à l'apprentissage de l'enfant ou à la scolarité, à l'éducation locale qui enseigne les "nuances "... autrement dit l'ensemble des références acquises au cours de l'éducation " (Laurian 2004), d'où la difficulté de l'exercice de traduction des expressions figées.

Notre contribution va se focaliser sur les locutions verbales figées telles que perdre le Nord, être au septième ciel, être aux anges, être hors de soi, sortir de ses gonds, s'arracher les cheveux... qui dénotent un état émotionnel. Ces expressions "renferment à la fois des éléments complètement figés et des éléments complètement libres : le noyau verbal connaît pratiquement toutes les variations morphologiques alors que les autres constituants sont souvent d'un emploi contraint" (Mejri 1997). Pour relever ces contraintes syntaxiques, distributionnelles et sémantiques, lors d'une approche "non-automatisée ", on passe nécessairement par une approche formelle ${ }^{8}$ (Leeman 2008). Mais comment s'opère cette reconnaissance au cours du traitement automatique de la langue et plus particulièrement par les logiciels de traduction automatique (TA) ?

\section{Les expressions figées et la traduction automatique}

Dans un contexte socio-économique de profusion, d'internationalisation et d'immédiateté, les logiciels de traduction répondent à un besoin croissant et pressant : traduire vite et moins cher. Parce qu'ils automatisent totalement ou partiellement le travail, ces logiciels sont aujourd'hui les partenaires privilégiés de la "localisation linguistique" ; ils ne se limitent donc plus aux logiciels de traduction automatique mais comprennent également les mémoires de traduction, les dictionnaires de traduction (bilingues ou multilingues), entre autres (Vaguer 2007). Nous regarderons donc quelle place est accordée à la traduction des expressions figées par/dans ces outils. Pour ce faire, nous avons retenu

\footnotetext{
Ainsi avons-nous mis en évidence (Vaguer 2005) que ces constructions, du fait de leur figement, bloquent une bonne partie des transformations (suppression, déplacements du GP, extraction...) que l'on utilise traditionnellement pour identifier des constructions dites «libres» mais que certains critères (la relativation, l'interrogation, l'interdiction d'un modifieur) s'avèrent déterminants pour la caractérisation de ces constructions verbales figées puisque à l'inverse des constructions à verbe prédicatif, celles-ci les interdisent :
}

(1) Nini croque dans une mangue.

a. La mangue que Nini croque.

b. Dans quoi croque-t-elle? - Dans une mangue.

c. Nini croque dans une mangue bien mûre.

(2) Léonard pédale dans la semoule.

a. * La semoule où Léonard pédale.

b. *(Où + Dans quoi) pédale-t-il ? - Dans la semoule.

c. * Léonard pédale dans la semoule cuite. 
deux logiciels de TA, libres d'accès - Systran et Reverso - auxquels nous avons soumis un corpus d'une quarantaine de locutions ${ }^{9}$, qui permettait d'observer la traduction des subtilités tant langagières que culturelles de la langue française. Nous en avons sollicité la traduction en anglais et en espagnol.

\subsection{Constats}

Par rapport aux corpus testés, les logiciels de TA offrent des performances peu satisfaisantes. Nos jugements ne porteront que sur la traduction des locutions verbales mais il y aurait beaucoup à dire sur la qualité générale des traductions obtenues (tant sur le plan syntaxique que lexical et sémantique).

40\% des locutions testées ne sont pas prises en charge par les logiciels de TA (i.e. aucun de deux logiciels n'est capable de proposer de traduction adéquate pour ces locutions). II s'agit des expressions perdre la boussole ; être sur des charbons ardents; être rouge comme un coq ; mettre hors de ses gonds; sortir de ses gonds ; s'arracher les cheveux; être le dos au mur ; être à ramasser à la petit cuiller ; en avoir plein (les bottes, le dos) ; en avoir sa dose (sa claque); chier (faire) dans son (froc, pantalon); se faire des cheveux; en baver; pleurer toutes les larmes de son corps, entre autres.

Les logiciels, pour pallier ces manques, proposent donc des traductions mot à mot qui ne sont pas pertinentes (cf. Tableau 1) :

\begin{tabular}{|l|l|l|}
\hline Locutions & Traductions EN & Traductions ES \\
\hline $\begin{array}{l}\text { perdre la boussole sur des charbons } \\
\text { ardents }\end{array}$ & 'lose the compass' & 'perder la brújula' \\
\hline être rouge comme un coq & 'red like a cock' & 'sobre carbones ardientes' \\
\hline mettre hors de ses gonds & $\begin{array}{l}\text { 'met out of his hinges' } \\
\text { 'put outside its hinges' }\end{array}$ & $\begin{array}{l}\text { 'pone fuera de sus bisagras } \\
\text { / goznes' }\end{array}$ \\
\hline s'arracher les cheveux & $\begin{array}{l}\text { 'tore off the hair' } \\
\text { 'tore away hair' }\end{array}$ & $\begin{array}{l}\text { 'arrancaban el cabello' } \\
\text { 'peleaban los cabellos' }\end{array}$ \\
\hline être le dos au mur & $\begin{array}{l}\text { 'to be the back with/in the } \\
\text { wall' }\end{array}$ & 'ser la espalda a la pared' \\
\hline $\begin{array}{l}\text { être à ramasser à la petite } \\
\text { cuiller }\end{array}$ & $\begin{array}{l}\text { 'to be collected with the } \\
\text { small spoon' ou 'in the } \\
\text { teaspoon' }\end{array}$ & 'recoger a la cucharilla' \\
\hline se faire des cheveux & 'to be made hair' & $\begin{array}{l}\text { 'hacerse del cabello' } \\
\text { 'hacerse cabellos' }\end{array}$ \\
\hline en baver & $\begin{array}{l}\text { 'to dribble about it' } \\
\text { 'to drool there' }\end{array}$ & $\begin{array}{l}\text { 'gotear' } \\
\text { 'babear de alli' }\end{array}$ \\
\hline
\end{tabular}

Tableau 1 - Locutions non prises en charge par les logiciels de TA

Les locutions qui sont prises en charge (60\% environ) - perdre les pédales; perdre le Nord; avoir le cœur qui bat la chamade; être heureux comme un poisson dans l'eau; être au septième ciel ; être aux anges; entrer (tomber) dans une colère noire ; être hors de soi ; être à bout (de nerfs); monter sur ses grands chevaux; être au bout du rouleau; en avoir ras le bol (le pompon, le cul, la casquette) ; être sur les dents ; faire froid dans le dos; avoir la chair

9 L'ensemble des traductions a été effectué en septembre 2009. 
de poule; se faire de la bile; retourner le couteau dans la plaie; fendre (crever) le cour ; rester bouche bée; avoir le cour gros, entre autres -, le sont :

- $\quad$ soit totalement (i.e. une traduction adéquate est proposée par le logiciel de TA dans les deux langues, en espagnol et en anglais), à $\mathbf{2 5 , 7 1} \%$ :

\begin{tabular}{|c|c|}
\hline être heureux comme un poisson dans l'eau & $\begin{array}{l}{[R]^{10} \text { to be as happy as a lark }} \\
{[R] \text { ser feliz como un pez en el }} \\
\text { agua }\end{array}$ \\
\hline être au septième ciel & $\begin{array}{l}\text { [S] to be in the seventh heaven } \\
\text { [S] estar en el séptimo cielo }\end{array}$ \\
\hline être aux anges & $\begin{array}{l}{[R] \text { to be in the seventh heaven }} \\
{[R] \text { estar en la gloria }}\end{array}$ \\
\hline $\begin{array}{l}\text { en avoir ras (le bol / le cul / le pompon / la } \\
\text { casquette) }\end{array}$ & $\begin{array}{l}\text { [S] to be fed up } \\
{[\mathrm{S}] \text { tener hastio }}\end{array}$ \\
\hline être sur les dents & $\begin{array}{l}{[R] \text { to be under great pressure }} \\
{[R] \text { andar de cabeza }}\end{array}$ \\
\hline avoir la chair de poule & $\begin{array}{l}{[S / R] \text { to have the gooseflesh }} \\
{[S / R] \text { tener carne de gallina }}\end{array}$ \\
\hline se faire de la bile & $\begin{array}{l}{[R] \text { to get worried about }} \\
{[R] \text { preocuparse }}\end{array}$ \\
\hline fendre le coeur & $\begin{array}{l}{[R] \text { to break you the heart }} \\
{[R] \text { partir el corazón }}\end{array}$ \\
\hline rester bouche bée & $\begin{array}{l}{[R] \text { open-mouthed }} \\
{[R] \text { boquiabierto }}\end{array}$ \\
\hline avoir le cœur gros & $\begin{array}{l}{[R] \text { to be in distress }} \\
{[R] \text { tener el corazón oprimido }}\end{array}$ \\
\hline
\end{tabular}

Tableau 2 - Locutions prises en charge en français et en espagnol par l'un des logiciels de TA

- $\quad$ soit partiellement (uniquement en anglais ou en espagnol par l'un des logiciels), à $34,29 \%$ :

\begin{tabular}{|l|l|}
\hline perdre les pédales & {$[\mathrm{R}]$ perder los estribos } \\
\hline perdre le Nord & $\begin{array}{l}{[\mathrm{S}] \text { no perder nunca el norte }} \\
{[\mathrm{R}] \text { perder la cabeza }}\end{array}$ \\
\hline avoir le cœur qui bat la chamade & $\begin{array}{l}{[\mathrm{S}] \text { to beating wildly }} \\
{[\mathrm{R}] \text { a heart pounding }}\end{array}$ \\
\hline être au septième ciel & {$[\mathrm{R}]$ estar en el séptimo cielo } \\
\hline $\begin{array}{l}\text { entrer (tomber) dans une colère noire (blanche } / \\
\text { bleue) }\end{array}$ & {$[\mathrm{R}]$ to be in a fury } \\
\hline être hors de soi & {$[\mathrm{S} / \mathrm{R}]$ estar fuera de sí } \\
\hline sembler (être) à bout (de nerfs) & {$[\mathrm{R}]$ at breaking point } \\
\hline
\end{tabular}

\footnotetext{
${ }^{10}[\mathrm{R}]$ désigne le logiciel de TA Reverso et [S] celui de Systran.
} 


\begin{tabular}{|l|l|}
\hline monter sur ses grands chevaux & {$[\mathrm{S}]$ to ride the high horse } \\
\hline être au bout du (de son) rouleau & {$[\mathrm{R}]$ to be exhausted } \\
\hline faire (avoir, donner) froid dans le dos & {$[\mathrm{R}]$ causar escalofrío } \\
\hline fendre (crever le cœur) & {$[\mathrm{S}]$ partir el corazón } \\
\hline
\end{tabular}

Tableau 3 - Locutions prises en charge dans l'une des langues par un des logiciels de TA

Ces observations permettent de conclure que le logiciel Systran ne prend en charge que partiellement les locutions verbales $\mathbf{7 1 , 4 3} \%$ des traductions proposées par le logiciel ne sont pas adéquates). Seules trois locutions sont traduites correctement dans les deux langues (être au septième ciel, avoir la chair de poule, en avoir ras le bol) et sept trouvent une seule traduction en langue cible (perdre le Nord, avoir le cour qui bat la chamade, être hors de soi, monter sur ses grands chevaux, remuer le couteau dans la plaie, fendre le cœur, avoir des trémolos dans la voix). On peut faire l'hypothèse que seules en avoir ras le bol, avoir le cœur qui bat la chamade, fendre le coeur ont été implémentées dans les ressources (dictionnaires) de Systran, pour les autres - être au septième ciel, avoir la chair de poule, être hors de soi... - on peut en douter car la traduction mot à mot ne pose ici aucun problème.

Reverso, en revanche, doit être identifié comme un logiciel de traduction qui intègre l'existence de ces locutions (54,29\% des traductions obtenues sont correctes) puisqu'il propose pour $22,86 \%$ d'entres elles (être heureux comme un poisson dans l'eau, être aux anges, être sur les dents, avoir la chair de poule, se faire de la bile, fendre le cour, rester bouche bée, avoir le cœur gros, entre autres), la traduction adéquate en anglais et en espagnol, et pour 31,43\% d'entre elles (perdre les pédales, perdre le Nord, être au septième ciel, entrer dans une colère noire, être hors de soi, être à bout (de nerfs), être au bout du rouleau, faire froid dans le dos, remuer le couteau dans la plaie, avoir des trémolos dans la voix, entre autres) une seule traduction (soit anglais, soit espagnol).

Conclusion. Il existe des logiciels de TA capables d'une part de reconnaître des locutions, d'autre part de les traduire. Mais tous ne prennent pas cette peine - ne procèdent pas à ce travail de reconnaissance et d'implémentation - car ce qu'ils privilégient c'est avant tout la quantité et non la qualité. Ainsi, on peut se satisfaire de cette évolution ${ }^{11}$, si minime soitelle.

Afin d'être encore plus pertinent, certains logiciels (Reverso) doivent renforcer l'implémentation des locutions dans leur dictionnaire et/ou ressources lexicales de base pour prendre en charge, notamment, leur traduction de façon bi-directionnelle. D'autres logiciels (Systran) ont à renforcer leur dictionnaire unilingue afin d'être en mesure de traduire bon nombre de lexèmes qui restent aujourd'hui non traduits. C'est donc avant tout un travail d'implémentation qui reste à mener par l'ingénieur linguiste car du point de vue de la reconnaissance des locutions, les logiciels sont capables de les identifier. Nous verrons (infra) que les lexiques multilingues que nous élaborons peuvent permettre à ces logiciels d'affiner, à moindre coût, leur traitement linguistique.

\subsection{Solutions envisagées}

Pour pouvoir traduire des locutions verbales, le recours à la traduction mot à mot doit être

11 En effet, E. Laporte (1988) mentionne que « les expressions figées posent des problèmes spécifiques en analyse automatique des textes en langues naturelles. Les systèmes existants ne les prennent pas en compte d'une façon satisfaisante, car on manque de méthodes générales pour les reconnaître ». 
abandonné. Il faut donc se tourner vers l'élaboration de lexiques multilingues (passant bien évidemment par des étapes intermédiaires, à savoir des lexiques bilingues) et la définition de règles de reconnaissance des dites locutions (qui permettent de les différencier d'une construction non figée).

\subsubsection{Elaboration de lexiques bilingues}

Il s'agit de répertorier l'ensemble des locutions verbales et leur équivalent dans les différentes langues : par exemple être heureux comme un poisson dans l'eau en anglais se traduit par to be happy as Larry / a sandboy / a clam / a lark (d'après le Robert \& Collins) : il faut donc "obtenir des correspondances entre les différentes dénominations des mêmes phénomènes, reposant chacune sur des stéréotypies propre à chaque langue; ce qui pourrait avoir un impact direct sur la solution des problèmes épineux de la traduction de ses séquences " (Mejri 1997).

Nous proposons dans le Tableau 4 une représentation de ce que pourrait être un lexique multilingue (anglais et espagnol) pour les locutions verbales étudiées :

\begin{tabular}{|c|c|c|c|}
\hline & Locutions verbales & [EN] & [ES] \\
\hline \multirow[t]{2}{*}{ Affolement } & $\begin{array}{l}\text { Perdre (la boussole / la } \\
\text { boule / la tête /les } \\
\text { pédales) }\end{array}$ & $\begin{array}{l}\text { to go off one's head } \\
\text { to lose one's marbles } \\
\text { to lose one's grip }\end{array}$ & $\begin{array}{l}\text { perder la cabeza } \\
\text { perder los estribos }\end{array}$ \\
\hline & Perdre le Nord & to lose one's way & $\begin{array}{l}\text { perder el norte } \\
\text { desnortarse }\end{array}$ \\
\hline \multirow[b]{2}{*}{$\begin{array}{l}\text { Angoisse, } \\
\text { Anxiété }\end{array}$} & $\begin{array}{l}\text { Avoir le cœur qui bat la } \\
\text { chamade }\end{array}$ & $\begin{array}{l}\text { His heart was pounding } \\
\text { / beating wildly }\end{array}$ & $\begin{array}{l}\text { su corazón se le salía } \\
\text { del pecho }\end{array}$ \\
\hline & $\begin{array}{l}\text { Etre sur des charbons } \\
\text { ardents }\end{array}$ & $\begin{array}{l}\text { to be like a cat on hot } \\
\text { bricks } \\
\text { to be like a cat on a hot } \\
\text { tin roof }\end{array}$ & estar en ascuas \\
\hline \multirow[t]{3}{*}{$\begin{array}{l}\text { Bonheur / Joie } \\
\text { / Gaité / Rire }\end{array}$} & $\begin{array}{l}\text { Etre heureux comme un } \\
\text { poisson dans l'eau }\end{array}$ & $\begin{array}{l}\text { to be happy as Larry } \\
\text { to be happy as a } \\
\text { sandboy } \\
\text { to be happy as a clam } \\
\text { to be happy as a lark }\end{array}$ & $\begin{array}{l}\text { sentirse como el pez } \\
\text { en el agua }\end{array}$ \\
\hline & Etre au septième ciel & $\begin{array}{l}\text { to be in seventh } \\
\text { heaven } \\
\text { to be on cloud nine }\end{array}$ & $\begin{array}{l}\text { estar en el séptimo } \\
\text { cielo }\end{array}$ \\
\hline & Etre aux anges & $\begin{array}{l}\text { to be in the seventh } \\
\text { heaven }\end{array}$ & estar en la gloria \\
\hline \multirow{4}{*}{ Colère, Rage } & $\begin{array}{l}\text { (Etre / Entrer / Tomber) } \\
\text { dans une colère (noire / } \\
\text { blanche / bleue) }\end{array}$ & $\begin{array}{l}\text { to flew into a terrible } \\
\text { rage } \\
\text { to be in a fury }\end{array}$ & $\begin{array}{l}\text { rabia impotente } \\
\text { cólera tremenda / } \\
\text { furibunda / furiosa }\end{array}$ \\
\hline & $\begin{array}{l}\text { Etre rouge comme un } \\
\text { coq }\end{array}$ & $\begin{array}{l}\text { to be as red as a } \\
\text { beetroot / a lobster }\end{array}$ & $\begin{array}{l}\text { estar encendido } \\
\text { como un pavo }\end{array}$ \\
\hline & Etre hors de soi & $\begin{array}{l}\text { to be beside o.s. (with } \\
\text { anger) }\end{array}$ & $\begin{array}{l}\text { estar fuera de sí } \\
\text { sacar a uno de sus } \\
\text { casillas }\end{array}$ \\
\hline & Etre (jeter, mettre) hors & to make sb wild with & sacar a uno de sus \\
\hline
\end{tabular}




\begin{tabular}{|c|c|c|c|}
\hline & Locutions verbales & [EN] & [ES] \\
\hline & de ses gonds & rage & casillas \\
\hline & Sortir de ses gonds & to fly off the handle & $\begin{array}{l}\text { salir uno de sus } \\
\text { casillas } \\
\text { salir de quicio }\end{array}$ \\
\hline & $\begin{array}{l}\text { (Etre / Pousser) à bout } \\
\text { (de nerfs) }\end{array}$ & $\begin{array}{l}\text { to have had enough } \\
\text { to be at the end of } \\
\text { one's patience } \\
\text { to be at the end of } \\
\text { one's tether } \\
\text { to be just about at } \\
\text { breaking point }\end{array}$ & $\begin{array}{l}\text { sacar a uno de sus } \\
\text { casillas }\end{array}$ \\
\hline & $\begin{array}{l}\text { (Monter / Etre) sur ses } \\
\text { grands chevaux }\end{array}$ & $\begin{array}{l}\text { to get on one's high } \\
\text { horse }\end{array}$ & subirse a la parra \\
\hline \multirow[t]{2}{*}{ Désespoir } & S'arracher les cheveux & $\begin{array}{l}\text { litt. To tear out one's } \\
\text { hair } \\
\text { litt. To pull out one's } \\
\text { hair } \\
\text { to tear one's hair out }\end{array}$ & $\begin{array}{l}\text { tirarse de los pelos } \\
\text { mesarse los cabellos }\end{array}$ \\
\hline & Etre (avoir) le dos au mur & $\begin{array}{l}\text { to have one's back to } \\
\text { the wall }\end{array}$ & $\begin{array}{l}\text { estar entre la espada } \\
\text { y la pared }\end{array}$ \\
\hline \multirow[t]{3}{*}{ Détresse } & $\begin{array}{l}\text { Etre au bout du (de son) } \\
\text { rouleau }\end{array}$ & $\begin{array}{l}\text { to be exhausted } \\
\text { to have come to the } \\
\text { end of the road } \\
\text { (mourir) }\end{array}$ & $\begin{array}{l}\text { no poder más } \\
\text { estar en las últimas } \\
\text { (près de mourir) }\end{array}$ \\
\hline & $\begin{array}{l}\text { Etre à ramasser à la } \\
\text { petite cuiller }\end{array}$ & $\begin{array}{l}\text { to be completely } \\
\text { shattered }\end{array}$ & estar hecho papilla \\
\hline & $\begin{array}{l}\text { (Demeurer / rester / Etre) } \\
\text { coi }\end{array}$ & $\begin{array}{l}\text { to remain silent } \\
\text { to be rendered } \\
\text { speechless }\end{array}$ & $\begin{array}{l}\text { no chistar } \\
\text { no decir esta boca es } \\
\text { mía }\end{array}$ \\
\hline \multirow{4}{*}{ Excédé } & $\begin{array}{l}\text { En avoir plein (les bottes/ } \\
\text { le cul / le dos) de qqch }\end{array}$ & $\begin{array}{l}\text { to be fed up with sth } \\
\text { to be sick and tired of } \\
\text { sth } \\
\text { to be pissed of with sth }\end{array}$ & $\begin{array}{l}\text { estar hasta los pelos } \\
\text { estar hasta la } \\
\text { coronilla } \\
\text { estar harto }\end{array}$ \\
\hline & $\begin{array}{l}\text { En avoir ras (le bol / le } \\
\text { cul / le pompon / la } \\
\text { casquette) }\end{array}$ & $\begin{array}{l}\text { to be fed up } \\
\text { to be sick to death of it } \\
\text { to be fed up to the } \\
\text { back teeth (with it all) }\end{array}$ & $\begin{array}{l}\text { estar hasta el gorro } \\
\text { estar hasta la } \\
\text { coronilla } \\
\text { tener hastio }\end{array}$ \\
\hline & $\begin{array}{l}\text { En avoir sa (dose / } \\
\text { claque) }\end{array}$ & $\begin{array}{l}\text { to be fed up } \\
\text { to have had more than } \\
\text { one's share of it } \\
\text { to fed up to the back } \\
\text { teeth } \\
\text { to fed up to the teeth }\end{array}$ & $\begin{array}{l}\text { estar hasta la } \\
\text { coronilla } \\
\text { estar hasta los pelos }\end{array}$ \\
\hline & Avoir les nerfs (en boule / & to be very tensed up / & estar hecho un \\
\hline
\end{tabular}




\begin{tabular}{|c|c|c|c|}
\hline & Locutions verbales & [EN] & [ES] \\
\hline & en pelote) & tense / edgy & manojo de nervios \\
\hline & Etre sur les dents & $\begin{array}{l}\text { to be under great } \\
\text { pressure }\end{array}$ & $\begin{array}{l}\text { andar de cabeza } \\
\text { estar reventado de } \\
\text { trabajo } \\
\text { no dar de sí }\end{array}$ \\
\hline & $\begin{array}{l}\text { (Pousser / Mettre) qqn à } \\
\text { bout }\end{array}$ & $\begin{array}{l}\text { to push sb to the limit } \\
\text { (of his patience) }\end{array}$ & sacar de sus casillas \\
\hline \multirow{8}{*}{ Peur, Frayeur } & $\begin{array}{l}\text { Chier (faire) dans son } \\
\text { (froc, pantalon) }\end{array}$ & to crap himself & morirse de miedo \\
\hline & $\begin{array}{l}\text { Avoir (donner, faire) froid } \\
\text { dans le dos }\end{array}$ & $\begin{array}{l}\text { to send shivers down } \\
\text { my spine }\end{array}$ & causar escalofrío \\
\hline & Avoir la chair de poule & $\begin{array}{l}\text { to make } s b \text { flesh creep } \\
\text { to give sb gooseflesh }\end{array}$ & tener carne de gallina \\
\hline & $\begin{array}{l}\text { Etre blanc comme un } \\
\text { linge }\end{array}$ & \multirow{5}{*}{$\begin{array}{l}\text { white as a sheet } \\
\text { white with fear } \\
\text { white as a ghost }\end{array}$} & blanco como el papel \\
\hline & $\begin{array}{l}\text { Etre blanc comme un } \\
\text { cachet d'aspirine }\end{array}$ & & \\
\hline & $\begin{array}{l}\text { Etre blanc comme un } \\
\text { drap }\end{array}$ & & \\
\hline & $\begin{array}{l}\text { Etre blanc comme un } \\
\text { cadavre }\end{array}$ & & \\
\hline & $\begin{array}{l}\text { Etre blanc comme un } \\
\text { mort }\end{array}$ & & \\
\hline \multirow[b]{2}{*}{ Souci } & Se faire de la bile & $\begin{array}{l}\text { to get worried (about) } \\
\text { worry o.s. sick (about) }\end{array}$ & $\begin{array}{l}\text { Preocuparse } \\
\text { Quemarse la sangre }\end{array}$ \\
\hline & $\begin{array}{l}\text { Se faire des cheveux } \\
\text { (blanc / gris) }\end{array}$ & to worry o.s. sick & $\begin{array}{l}\text { quemarse la sangre } \\
\text { preocuparse } \\
\text { inquietarse }\end{array}$ \\
\hline \multirow{3}{*}{ Souffrance } & $\begin{array}{l}\text { (Enfoncer, remuer, } \\
\text { retourner) le couteau } \\
\text { dans la plaie }\end{array}$ & $\begin{array}{l}\text { to twist the knife in the } \\
\text { wound } \\
\text { to rub it in }\end{array}$ & $\begin{array}{l}\text { avivar la herida } \\
\text { herir en carne viva }\end{array}$ \\
\hline & En baver & $\begin{array}{l}\text { to have a rough } \\
\text { to have a hard time of } \\
\text { it }\end{array}$ & $\begin{array}{l}\text { reventar } \\
\text { parsarlas negras }\end{array}$ \\
\hline & (Fendre / Crever) le cœur & $\begin{array}{l}\text { to break sb heart } \\
\text { to make sb heart bleed } \\
\text { heartbreaking } \\
\text { heartrending }\end{array}$ & partir el corazón \\
\hline Stupéfaction & $\begin{array}{l}\text { (Etre / rester) bouche } \\
\text { (bée / béante) }\end{array}$ & $\begin{array}{l}\text { litt. to stand open- } \\
\text { mouthed } \\
\text { litt. to stand gaping } \\
\text { His jaw dropped / He } \\
\text { was flabbergasted (il } \\
\text { en est resté bouche }\end{array}$ & $\begin{array}{l}\text { quedarse } \\
\text { boquiabierto }\end{array}$ \\
\hline
\end{tabular}




\begin{tabular}{|c|c|c|c|}
\hline & Locutions verbales & [EN] & [ES] \\
\hline & & bée) & \\
\hline & Les bras m'en tombent & $\begin{array}{l}\text { I'm flabbergasted } \\
\text { I'm stunned }\end{array}$ & $\begin{array}{l}\text { Me quedo de une } \\
\text { pieza }\end{array}$ \\
\hline \multirow[t]{2}{*}{ Tristesse } & Avoir le coeur gros & $\begin{array}{l}\text { to have a heavy heart } \\
\text { to be in distress }\end{array}$ & $\begin{array}{l}\text { tener el corazón } \\
\text { oprimido, hecho } \\
\text { polvo }\end{array}$ \\
\hline & $\begin{array}{l}\text { Pleurer toutes les larmes } \\
\text { de son corps }\end{array}$ & to cry one's eyes out & $\begin{array}{l}\text { estar hecho un mar } \\
\text { de lágrimas }\end{array}$ \\
\hline Emotion & $\begin{array}{l}\text { Avoir des trémolos dans } \\
\text { la voix }\end{array}$ & $\begin{array}{l}\text { with a quaver } \\
\text { with tremor in one's } \\
\text { voice }\end{array}$ & temblor \\
\hline
\end{tabular}

Tableau 4 - Extrait d'un lexique multilingue de locutions élaboré par C. Vaguer

L'élaboration de ce type de lexique, s'il permet de prendre en compte la traduction des locutions en vue d'une implémentation informatique, offre également des données pour l'apprentissage d'une langue étrangère (car du fait que ces expressions traduisent une culture, il faut aider les apprenants à s'en imprégner à l'aide d'outils, comme les lexiques envisagés ici).

Ces index multilingues pourraient également servir de base à la préparation de dictionnaires bilingues comme aide aux lexicographes dans leur travail quotidien, mais aussi comme aide pour le traducteur. Cela reste un idéal, certes, mais que nous aimerions approcher, car comme le signale G. Gréciano (2003) " bien des fois, le caractère insuffisant, voire le déficit locutionnel des dictionnaires généraux traditionnels a été regretté : formes désuètes, ni représentatives, ni exhaustives, ni conviviales ". D'où la nécessité de nos travaux de recherche, de l'élaboration de nos glossaires (ressources lexicales) pour tenter d'y remédier.

\subsection{2. Élaboration de règles " cotextuelles "}

Une fois le lexique bilingue établi, il s'agit d'introduire au sein des analyseurs syntaxiques (le poumon de ces logiciels !) une règle que l'on peut qualifier de "cotextuelle ". Décrire la distribution (i.e. ce qui est dans le contexte gauche ou droit de perdre, avoir, être, entrer, mettre, sortir, monter...) va se trouver déterminant pour désambiguïser l'emploi de ces verbes - de leur emploi purement prédicatif à leur emploi figé - et pour traduire en conséquence. En effet, si on a une même forme verbale en français pour ces deux types d'emploi - pédaler dans (le vide + la semoule), tomber dans (le vide + les pommes), avoir un chat (dans la maison + la gorge), avoir des fourmis dans (la maison + les jambes), poser (un tableau + un lapin), prendre (le train + le taureau par les cornes), secouer (son oreiller + les puces à qqn) - ce n'est le cas ni en espagnol ni en anglais (cf. les erreurs de traduction obtenues supra). Ainsi, on peut indiquer d'ores et déjà comment - au moins pour certaines expressions - distinguer entre figé et non figé. Par exemple : tomber dans les pommes en tant qu'expression figée ne peut avoir qu'un sujet humain, donc un énoncé comme la cerise est tombée dans les pommes peut recevoir une traduction 'littérale' (tomber dans les pommes ne saurait ici signifier "s'évanouir "); de même pour rouler dans la farine, mais cette fois-ci c'est le complément d'objet qui ne peut être qu'humain : rouler les filets de poisson dans la farine ne peut donc avoir qu'une traduction littérale (et inversement pour 
rouler quelqu'un dans la farine); enfin avoir des fourmis dans les jambes ne peut guère avoir que l'interprétation figurée, sinon on dirait avoir des fourmis sur les jambes. Tout comme avoir le moral dans les chaussettes ne peut être ambigu et peut donc être enregistré 'en bloc'.

\section{Conclusion}

Les études sur le figement " ont permis de mettre en évidence le fait qu'on est en présence d'un des phénomènes les plus importants des langues naturelles, qui présente, pour le traitement automatique, un obstacle aussi important que la polysémie, par exemple " (Clas $\&$ Gross, 1998). Il faut donc répertorier l'ensemble de ces emplois, étudier leur distribution, afin de pouvoir définir des règles (qu'est-ce qui dans le contexte mais aussi, simplement, dans la forme même de la séquence, la signale comme expression figée et va permettre de lever l'ambiguïté ?).

La traduction des locutions reste problématique bien que leur usage soit loin d'être marginal, notamment en français. Si les logiciels de TA peuvent les prendre en charge (i.e. au-delà de la traduction mot à mot, des équivalences ${ }^{12}$ ont été établies entre les langues) cela ne s'opère pas de façon systématique. Pourquoi ? Qu'est-ce qui fait qu'une locution sera implémentée ou non dans les dictionnaires / ressources des logiciels ? Leur fréquence d'emploi ? Des demandes particulières de la part des clients ?

II devient donc nécessaire de procéder à des études comparées systématiques pour qui s'intéresse au traitement automatique des langues. Les conclusions des travaux spécialisés sur l'étude de séquences figées ne peuvent plus rester sur des constats identiques: " ce serait tout de même bien le diable si, à la suite d'études descriptives [...], on ne finissait pas par mobiliser des équipes pluridisciplinaires (linguistes, sociologues, anthropologues, informaticiens) pour s'atteler à ce travail d'articulation d'informations à la fois morphosyntaxiques, sémantiques, historico-culturelles et symboliques, qui sont indispensables à la traduction et à la rédaction assistées par ordinateur!" (Cortès 2004), "il serait du plus haut intérêt que des lexiques-grammaires bilingues d'expressions figées soient développés » (Danlos 1988). Où est l'implémentation des données descriptives ? Où sont ces lexiques ? S'ils existent, pourquoi ne sont-ils pas mis en valeur et exploités de façon significative dans les logiciels de TA ? Pourquoi en France les recherches universitaires n'attirent pas plus les développeurs de logiciels qui connaîtraient des avancées considérables en matière de prise en charge de la langue ? Nous laissons la réflexion en suspens, mais aimerions à mon tour que la recherche universitaire connaisse des débouchés industriels...

\section{Références}

ANDRIENIERENANA, C.-L. et al. : "Problème de traduction des expressions figées : un exemple bilingue français-malgache, malgache-français" dans Actes du Colloque international "Traduction humaine, Traduction automatique, Interprétation ", Série Linguistique n¹1, 2000.

BERCKER, François: Étude comparative anglais-français des expressions figées figurées comportant un ou plusieurs noms de parties du corps humain, Thèse de l'Université Paris III Sorbonne Nouvelle, 1994.

BERCKER, François : "La référence au corps humain dans les expressions figées figurées »

12 Ce qui prouve que le figement est un phénomène propre à chaque langue puisqu'il ne s'effectue pas forcément avec les mêmes structures, ni le même lexique. 
dans Travaux linguistiques du Cerlico, 12, 1999, p. 269-289.

BIDAUD, Françoise : Structures figées de la conversation. Analyse contrastive français-italien, Études contrastives, 4, Berne : Peter Lang, 2002.

BILlAUDEAU, Armand Georges: Recueil de locutions françaises proverbiales, familières, figurées traduites par leurs équivalents anglais, Paris : Boyveau et Chevillet, 1903.

BLANCO, Xavier: "Les déterminants nominaux figés. Perspective contrastive espagnolfrançais » dans S. Mejri, G. Gross, A. Clas \& T. Baccouche (éds) : Le figement lexical, Tunis : CERES, 1998, p. 19-34.

BLANCO, Xavier (éd): Langages $\mathrm{n}^{\circ} 143$ : Lexicologie contrastive espagnol-français, Paris: Larousse, 2001.

CALVET, Louis-Jean : Pour une écologie des langues du monde, 1999, Paris : Plon.

CLAS, André \& GROSS, Gaston : "Classes de figement des locutions verbales » dans S. Mejri, G. Gross, A. Clas \& T. Baccouche (éds) : Le figement lexical, Tunis : CERES, 1998, p. 11-18.

ConenNA, Mirella: "Les expressions 'figées' en français et en italien : problèmes lexicosyntaxiques de traduction » dans Contrastes, 10, 1985, p. 129-144.

CORTES, Colette : «Nombres symboliques, nombres diaboliques! De la subjectivité des opérations de quantification, de qualification et de classement dans la phraséologie des nombres en allemand et en français " dans A.-M. Laurian (éd) : Dictionnaires bilingues et interculturalité, 2004, p. 85-140.

DANLOS, Laurence : "La morphosyntaxe des expressions figées " dans Langages, 63, 1981, p. 53-74.

DANLOS, Laurence (éd) : Langages nْ90 : Les expressions figées, Paris : Larousse, 1988.

DUBoIs, Jean \& DUBOIS-CHARLIER, Françoise : Locutions en français, Aix-en-Provence : chez les auteurs, 2004.

DuBoıs, Marguerite-Marie: Dictionnaire anglais-français des locutions et expressions verbales, Paris : Larousse, 1973.

DugAs, André \& Di-ScIullo, Anna Maria: "Le traitement lexical des phrases figées du français, de l'espagnol et de l'italien " dans Actes du XVII Congrès international de linguistique et de philologie romanes, Aix en Provence, 1978.

GAATONE, David : " La locution : analyse interne et analyse globale » dans M. Martins-Baltar (éd) : La locution : entre langue et usages, 1997, p. 165-177.

GARCIA-PELAYO, Ramón et al. : Gran diccionario español-francés, francés-español, 1998, Paris : Larousse.

GIRY, Jacqueline: "Jean fait le (généreux + le diable). Constructions productives et expressions figées " dans Revue québécoise de linguistique, 13 : 2, 1984, p. 216-236.

GOUADEC, Daniel : Profession traducteur, Éd. La Maison du Dictionnaire, 2002.

GoUGENHEIM, Georges : "Une catégorie lexico-grammaticale : les locutions verbales » dans Études de linguistique appliquée, 2, 1971, p. 56-64.

GRÉCIANO, Gertrud : "Phraséologie et traduction » dans S. Mejri, T. Baccouche, A. Clas \& G. Gross (éds) : Traduire la langue, Traduire la culture, Sud Éditions, Paris: Maisonneuve et Larose, 2003, p. 81-93.

Gross, Gaston : Les expressions figées en français: noms composés et autres locutions, Paris/Gap : Ophrys, 1996.

Gross, Maurice : « Une classification des phrases 'figées' du français » dans Revue québécoise de linguistique, $11: 2,1982$, p. 151-185.

Gross, Maurice : "Sur les déterminants dans les expressions figées » dans Langages, 79, 1985, p. 89-117. 
Gross, Maurice : Grammaire transformationnelle du français. 3. Syntaxe de l'adverbe, Paris : ASSTRIL, 1986a.

Gross, Maurice : "Les nominalisations d'expressions figées » dans Langue française, 69, 1986b, p. 64-84.

Gross, Maurice : « Les limites de la phrase figée » dans Langages, 90, 1988a, p. 7-22.

GRoss, Maurice : "Sur les phrases figées complexes du français » dans Langue française, 77, 1988b, p. 47-70.

Gross, Maurice : "Les phrases figées en français » dans L'information grammaticale, 59, 1993, p. 36-41.

HAGÈGE, Claude : L'homme de parole. Contribution linguistique aux sciences humaines, 1987, Paris : Gallimard.

HAUSMANN, Franz Josef : "Tout est idiomatique dans les langues " dans M. Martins-Baltar (éd) : La locution : entre langue et usages, 1997, p. 277-290.

HUMBLEY, John : "Approches définitoires du rapport culturel français/autre langue dans les dictionnaires spécialisés bilingues " dans A.-M. Laurian (éd) : Dictionnaires bilingues et interculturalité, 2004, p. 163-181.

LABELLE, Jacques : "Lexiques-grammaires comparés : formes verbales figées en français du Québec »dans Langages, 90, 1988, p. 73-97.

LAPORTE, Éric: "La reconnaissance des expressions lors de l'analyse automatique " dans Langages, 90, 1988, p. 117-126.

LAURIAN, Anne-Marie (éd): Dictionnaires bilingues et interculturalité, Berne: Peter lang, 2004.

LEEMAN, Danielle (éd) : L'information grammaticale n¹17 : Les locutions prépositionnelles en 'sous', Paris : Peeters, 2008.

MARTINS-BALTAR, Michel \& CALBRIS, Geneviève (éds) : Le corps dans la langue, Lexicographica, Tübingen : Niemeyer, 1997.

MEJRI, Salah: Le figement lexical. Descriptions linguistiques et structuration sémantique, Publications de la Faculté des Lettres de la Manouba, 1997.

MEJRI, Salah : "Figement et formation d'outils syntaxiques " dans Travaux linguistiques du Cerlico, 14, 2001, p. 203-214.

[Reverso] http://www.reverso.com/text translation.asp

SELESKOVITCH, Danica : Interpréter pour traduire, Paris : Didier, 1984.

[Systran] http://www.systranet.com/systran/net

VAguer, Céline: "Pédaler dans la semoule. Approches des constructions verbales figées de structures 'V dans GN' » dans Linx, 53, 2005, p. 231-245.

VAGUER, Céline : "Expressions figées et traduction : de la langue aux outils ", Communication au Colloque International 'Traduction et communication interculturelle' (29 septembre au 1 octobre 2006), Sofia, Bulgarie, 2006.

VAGUER, Céline: "Panorama des outils d'aide à la traduction automatique ", Communication, Salon Expolangues (Paris) dans le cadre de la Table ronde Les nouveaux outils de la traduction : gadget ou révolution culturelle ? organisée par le Ministère de la Culture et de la communication et la Délégation Générale à la Langue Française et aux Langues de France (DGLFLF), 2007.

VAGUER, Céline: "Locutions verbales 'être doux comme un agneau' et traduction. Langue, culture et traduction automatique ", dans J.-C. Anscombre (dir.) : Recueil sur le figement, à paraître.

VAGUER, Céline : "Locutions prépositionnelles en sous : classement syntaxique, ressources 
lexicales, traduction automatique » dans Le français moderne, 2010.

VALLI, André \& VILAGINES SERRA, Eulalia : "Locutions figées comprenant un nom 'partie du corps' en espagnol et en français » dans S. Mejri, G. Gross, A. Clas \& T. Baccouche (éds) : Le figement lexical, Tunis : CERES, 1998, p. 177-206.

Van Hoof, Henri : Un Monde de langues. Petit panorama à l'usage du profane, 2009, Genève : Éditions Slatkine.

VARROD, P. et coll. : Le Robert \& Collins Super Señor, Paris : Dictionnaires Le Robert, $2000^{2}\left(1^{\text {re }}\right.$ éd. 1995).

\section{Biographie :}

Céline Vaguer est Maître de conférences en Sciences du langage à I'Université de Toulouse II-Le Mirail. Au sein du Département de Lettres modernes, Théâtre et Occitan, elle dispense des cours de syntaxe, d'introduction à la linguistique, de phonologie, de préparation à l'Agrégation de Lettres (classiques et modernes) et de valorisation de la recherche, entre autres. Rattachée au laboratoire CLLE-ERSS (UMR 5263), ses recherches portent principalement sur la langue française dans une perspective synchronique, mais elles connaissent également des approches comparatives inter-langues et diachroniques. Elles ont pour point de départ le domaine de la syntaxe et se centrent sur l'étude de mécanismes syntaxiques, sans pour autant s'y restreindre puisque qu'elles sont mâtinées de lexique et de sémantique. Elles connaissent diverses perspectives tant en linguistique française qu'en didactique ou du point de vue de la modélisation des données. 